\title{
Investigation of natural frequencies of axially loaded thin-walled columns
}

\author{
Lukasz Żmuda-Trzebiatowski ${ }^{1{ }^{*}}$ \\ ${ }^{1}$ Gdańsk University of Technology, Faculty of Civil and Environmental Engineering, \\ ul. Narutowicza 11/12, 80-233 Gdańsk, Poland
}

\begin{abstract}
The paper deals with correlation between natural frequencies of two steel thin-walled columns and the corresponding applied load. The structures are made of cold-formed lipped channel sections. The columns lengths were assumed to follow two buckling patterns global flexural and flexural-torsional buckling. In the thicker structure two material models were considered - linearly-elastic and elastic-perfectly plastic. Numerical computations cover dynamic eigenvalue problem, linear buckling and geometrically (and materially) non-linear analysis. The correlation between squares of natural frequencies and the applied load is linear in both columns. The first natural frequencies drop to zero due to structural buckling. This method, called the Vibration Correlation Technique, allows to predict buckling loads on the basis of measured vibration frequencies of the structures. Plasticity does not affect the corresponding curves - the use of the presented technique is limited to the structures exhibiting elastic buckling behaviour.
\end{abstract}

\section{Introduction}

Cold-formed thin-walled profiles are widely used in civil engineering, e.g. as elements strengthening silos or girts or purlins in roofs and wall structures. They exhibit three kinds of stability loss - local, distortional and global [1]. The latter can be flexural, torsional or a combination of both. The actual buckling mode is affected by material data and a slenderness ratio. The local buckling behaviour is linked with a small slenderness ratio. The slenderness ratio increase makes the structures exhibit distortional stability loss, next triggering global buckling modes. The buckling load in thick compressed elements is relatively large, therefore the limit load exceeds an elastic-range stability problem. Elastic buckling is observable in elements of a high slenderness ratio.

The prediction of realistic buckling loads of compressed thin-walled elements by means of experimental tests can be useful for the validation of numerical models of new or existing structures. One of the non-destructive methods allowing to determine the buckling load of structures is the Vibration Correlation Technique (VCT) [2]. It assumes linear correlation between compressive load and squares of natural frequencies for a majority of steel structures:

\footnotetext{
*Corresponding author: luktrzeb@pg.edu.pl
} 


$$
\left(f_{\mathrm{n}} / f_{\mathrm{n} 0}\right)^{2}=1-\left(P / P_{\mathrm{n}}\right)
$$

where:

$f_{\mathrm{n}} \quad n$th natural frequency of the loaded structure;

$f_{\mathrm{n} 0} \quad n$th natural frequency of the unloaded structure;

$P \quad$ applied load;

$P_{\mathrm{n}} \quad$ buckling load corresponding to $n$th vibration mode.

While a structure experiences buckling, the first natural frequency tends to zero. Experimental tests show that this tendency is not always apparent - sometimes it reaches its minimum, next rises again [3].

Thus, recording acceleration signals of structures at two loading stages, next making a time-to-frequency domain the Fourier transform, it is possible to predict primary buckling loads. Such a technique can improve the safety of structural steel and composite bucklingprone structures.

Nevertheless, some shell structures, e.g. column-strengthened silos, are characterised by a non-linear load-frequency squared relationship [4]. The relation is linear only at the start; the second half shows it curvilinear leading to the predicted buckling greater than the realistic one.

In the literature the VCT has been mainly used in panels and cylindrical shells $[5,6]$. The paper covers an application of the Vibration Correlation Technique to cold-formed thin-walled profiles exhibiting global buckling.

\section{Investigated structures}

\subsection{Description of the columns}

The effectiveness of the presented method was verified on two examples of thin-walled columns. The height of the first structure (A) was $9000 \mathrm{~mm}$, the height of the second (B) was $2500 \mathrm{~mm}$. In both cases the length was assumed to follow different buckling modes both global, A - flexural, B - flexural-torsional. The columns were made of the same coldformed lipped $4 \mathrm{~mm}$ thick channel section. The dimensions of the cross-section are given in Fig. 1a. The material is steel S235 with following parameters: Poisson's ratio $v=0.3$, elasticity modulus $E=210 \mathrm{GPa}$, specific weight $\rho=7850 \mathrm{~kg} / \mathrm{m}^{3}$ and yield stress $f_{\mathrm{y}}=235 \mathrm{MPa}$.

\subsection{Numerical models}

The columns were modelled in the FEM-based ABAQUS software [7]. In both (A) and (B) models 4-node shell elements with reduced integration were applied. The FEM model of the column A consists of 41'152 elements, while the column B model consists of 22'000 elements.

The material model in the higher column exhibiting global flexural buckling (A) was set linearly-elastic due to the limit load much higher than the minimum buckling load. The second column height to trigger the influence of plasticity greater compared to stability impact. Therefore, the structure B covers two material models - linearly-elastic and elasticperfectly plastic.

The bottom edges were fixed at both columns. Translations in all directions and rotations about all axes at top edges were constrained, except the vertical deflections (corresponding to the column longitudinal axes). 
The load was applied vertically to the upper structural edges in order to provide axial compression. The column model view is shown in Fig. $1 \mathrm{~b}$.
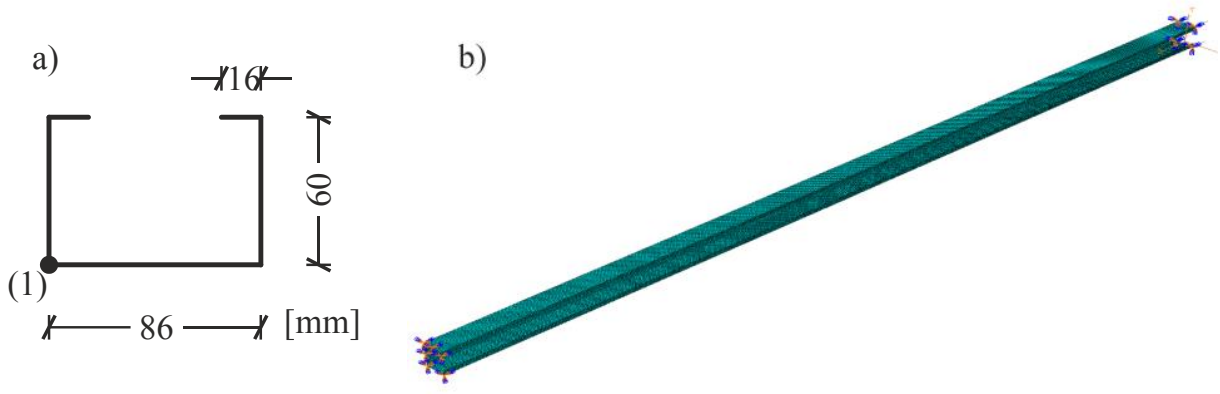

Fig. 1. The column cross-section (a) and the numerical model (b).

Numerical investigation of thin-walled columns covers dynamic eigenvalue problem, linear buckling and geometrically (and materially) non-linear analysis. Linear buckling analysis was performed in order to obtain the primary five buckling loads and the corresponding modes. The static, general method with a load as a control parameter was used to consider the cases of non-linear analysis. The initial, minimum and maximum increment sizes were equal to $0.001,10^{-30}$ and 0.003 , respectively. Geometrically non-linear analysis led to full equilibrium paths showing column load versus vertical displacement of a node 1 in a corner of the channel section in the upper edges of the structures (see Fig. 1a). Additionally, automatic stabilization in a form of a damping factor was introduced to provide solution convergence. The damping factor was equal to $10^{-8}$ in the column $\mathrm{A}$ and $10^{-7}$ in the column B. Dynamic eigenvalue analysis was conducted to find the primary natural frequencies and the corresponding vibration modes.

Geometrically (and materially) non-linear analysis and dynamic eigenvalue analysis were performed repeatedly. In each course the load increased from zero to a prescribed level in the non-linear analysis case in order to pre-stress the columns. Next, dynamic eigenvalue analysis was conducted. On the basis of the performed analysis the relationships between the squares of natural frequencies and the applied compressive loads were plotted, illustrating the conducted experiments.

\section{Results}

\subsection{Column A}

The evolution of maximum compressive forces in the column A against vertical displacement of the reference node resulting from geometrically non-linear analysis was plotted in Fig. 2a. The maximum load carrying-capacity was equal to $48.5 \mathrm{kN}$. The structure deformation produced by the applied load at its limit point exhibits global, flexural deformation shape (Fig. 2b). The limit load equals $212 \mathrm{kN}$, thus it does not act on stability loss in any way.

The first buckling load in the column A equals $43 \mathrm{kN}$. The corresponding buckling mode shows a global, flexural deformation shape including a single semi-wave. The deformation patterns in both static non-linear and buckling analysis converge. The second buckling load equals $59.8 \mathrm{kN}$, the corresponding buckling mode shows a flexural-torsional mode. The primary five buckling modes and their corresponding buckling loads are given in Fig. 3a. The Euler's buckling load with respect to the weak axis equals $43 \mathrm{kN}$. 
a)

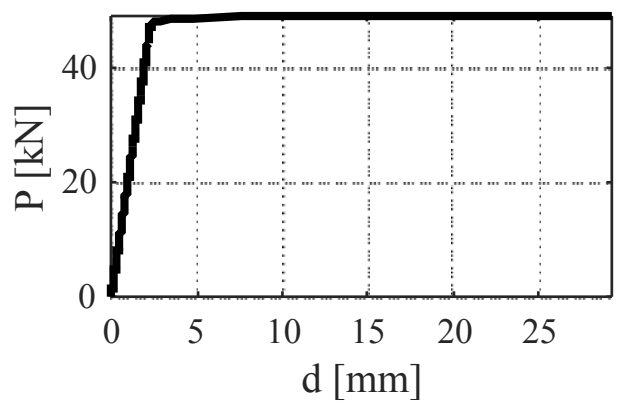

b)

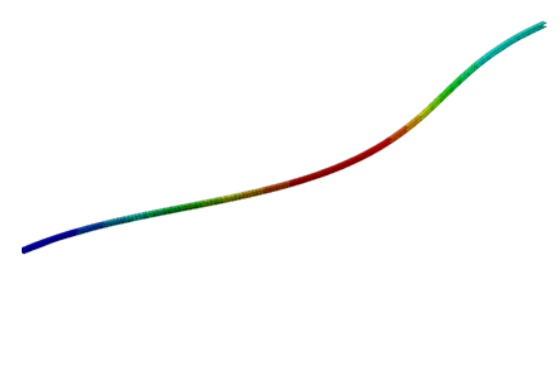

Fig. 2. The evolution of maximum compressive forces and vertical displacement of the node 1 in the column A obtained from static non-linear analysis (a) and the structure deformation (b).
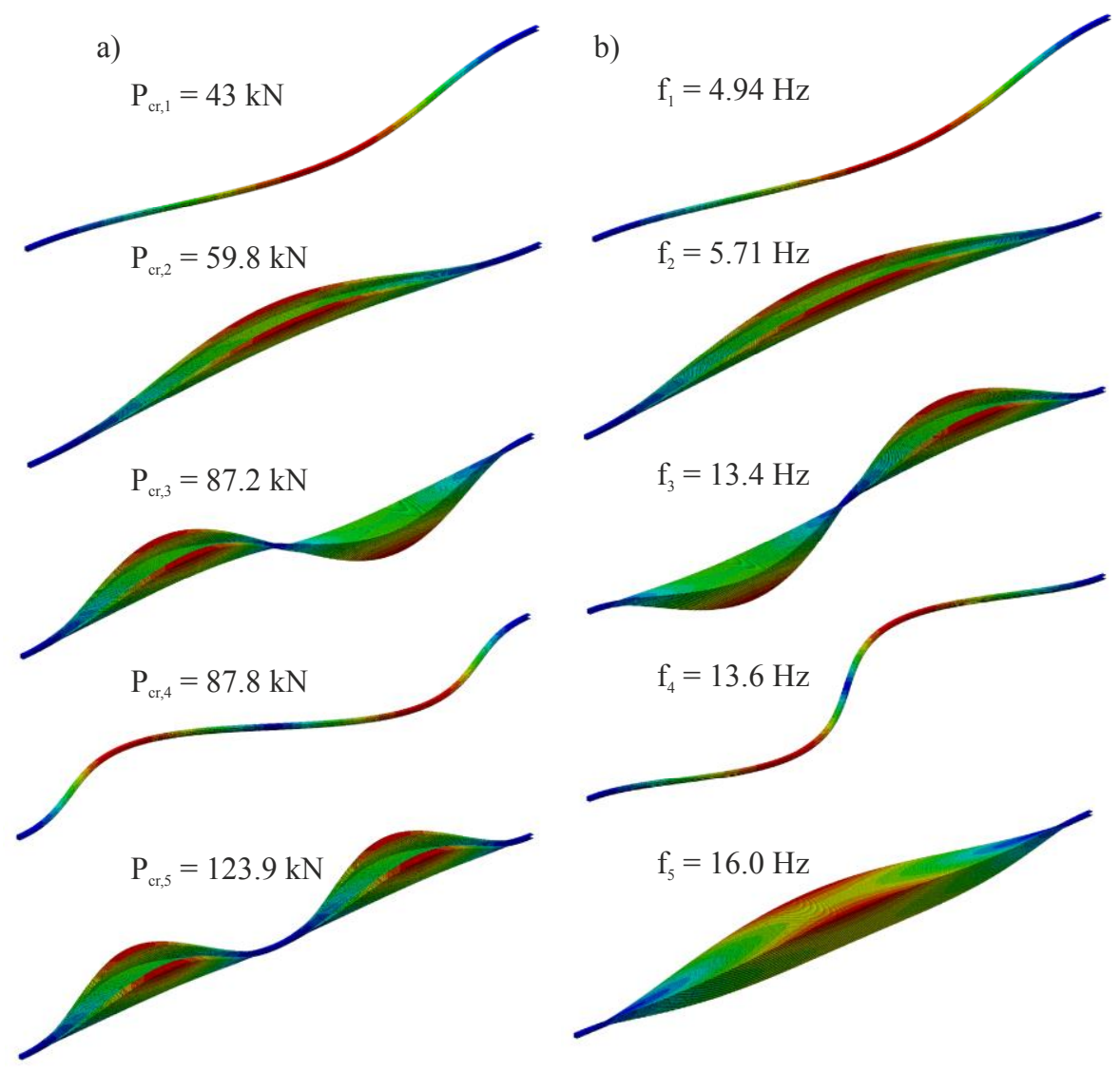

Fig. 3. The primary modes in the column A: linear buckling analysis (a) and dynamic eigenvalue analysis (b).

The first natural frequency equals $4.94 \mathrm{~Hz}$, the second $-5.71 \mathrm{~Hz}$. The primary five vibration modes and their corresponding natural frequencies are presented in Fig. 3b. The buckling and vibration one-to-four modes resemble, the fifth modes diverge. 


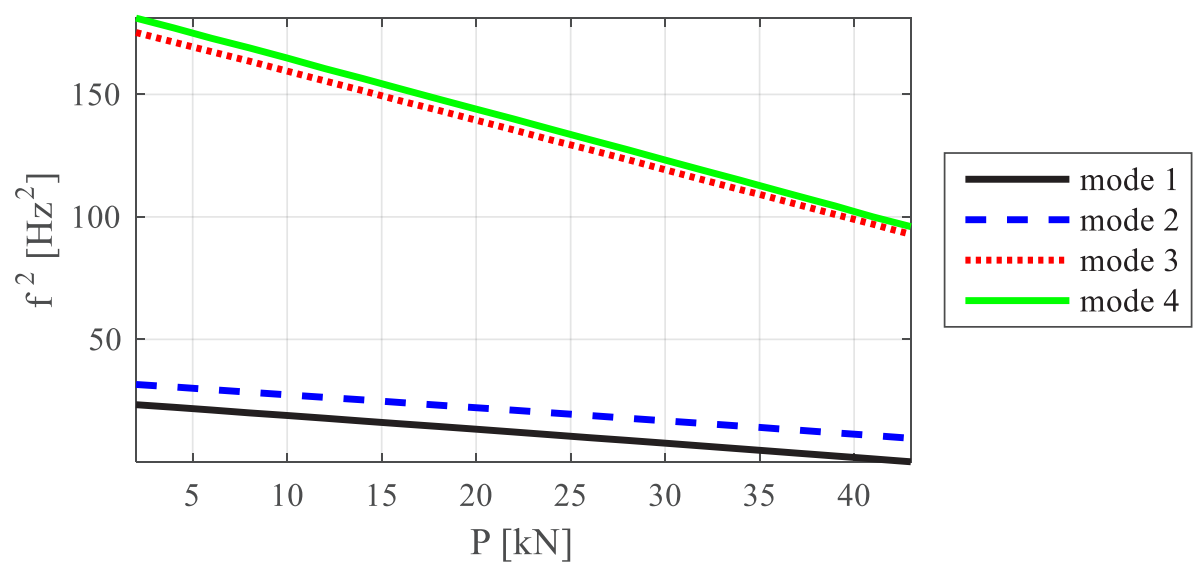

Fig. 4. The correlation between compressive load and squares of natural frequencies in the column A.

The correlation between the compressive forces and the squares of the first four natural frequencies was plotted in Fig. 4. Linear relations occur in each case - the lowest square of a correlation coefficient is 0.9999 . The first natural frequency drops to zero when the load equals $43 \mathrm{kN}$ - the exact value of the first buckling load. The Vibration Correlation Technique seems to work sufficiently well in the range of thin-walled compressed elements.

\subsection{Column B}

The evolution of maximum compressive forces in the column B against vertical displacement of the reference node resulting from the geometrically non-linear analysis was plotted in Fig. 5a. The maximum compressive load carrying-capacity is equal to $430.2 \mathrm{kN}$. The structure deformation caused by the applied load at its limit point shows a global, flexural-torsional deformation (Fig. 6a). Application of the damping factor $10^{-8}$ or omitting the solution stabilization result in a different deformation shape - still global, but flexural. Thus the maximum compressive carrying-capacity equals $555 \mathrm{kN}$, showing a $29 \%$ increase.

a)

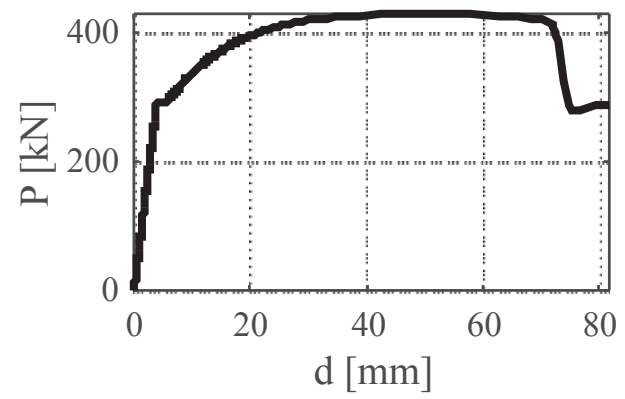

b)

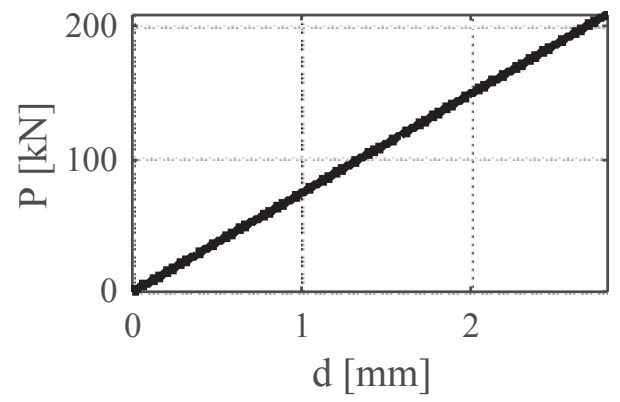

Fig. 5. The evolution of maximum compressive forces and vertical displacement of the node 1 in the column B obtained from static non-linear analysis with linearly-elastic (a) and elastic-perfectly plastic material models (b).

Numerical model of column B, employing the elastic-perfectly plastic material model shows the maximum limit load equal $212 \mathrm{kN}$. The corresponding equilibrium path and deformation shape are shown in Figs. $5 b$ and $6 \mathrm{~b}$. 


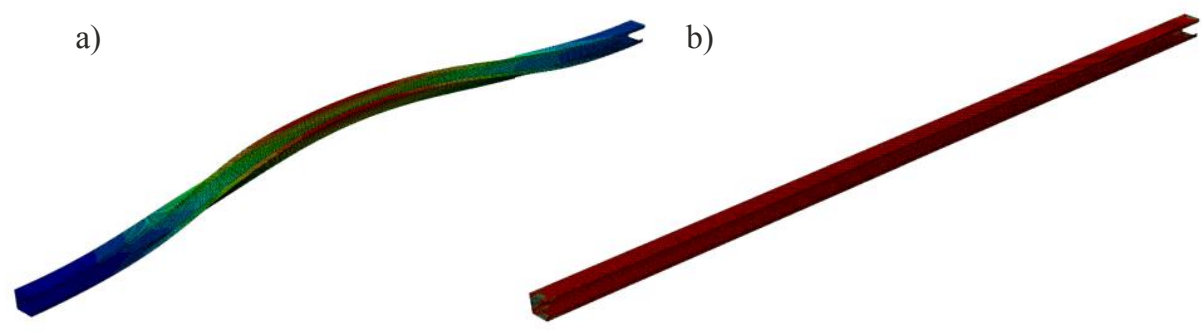

Fig. 6. The column B deformation at the time of the loss of stability with linearly-elastic (a) and elastic-perfectly plastic material models (b).

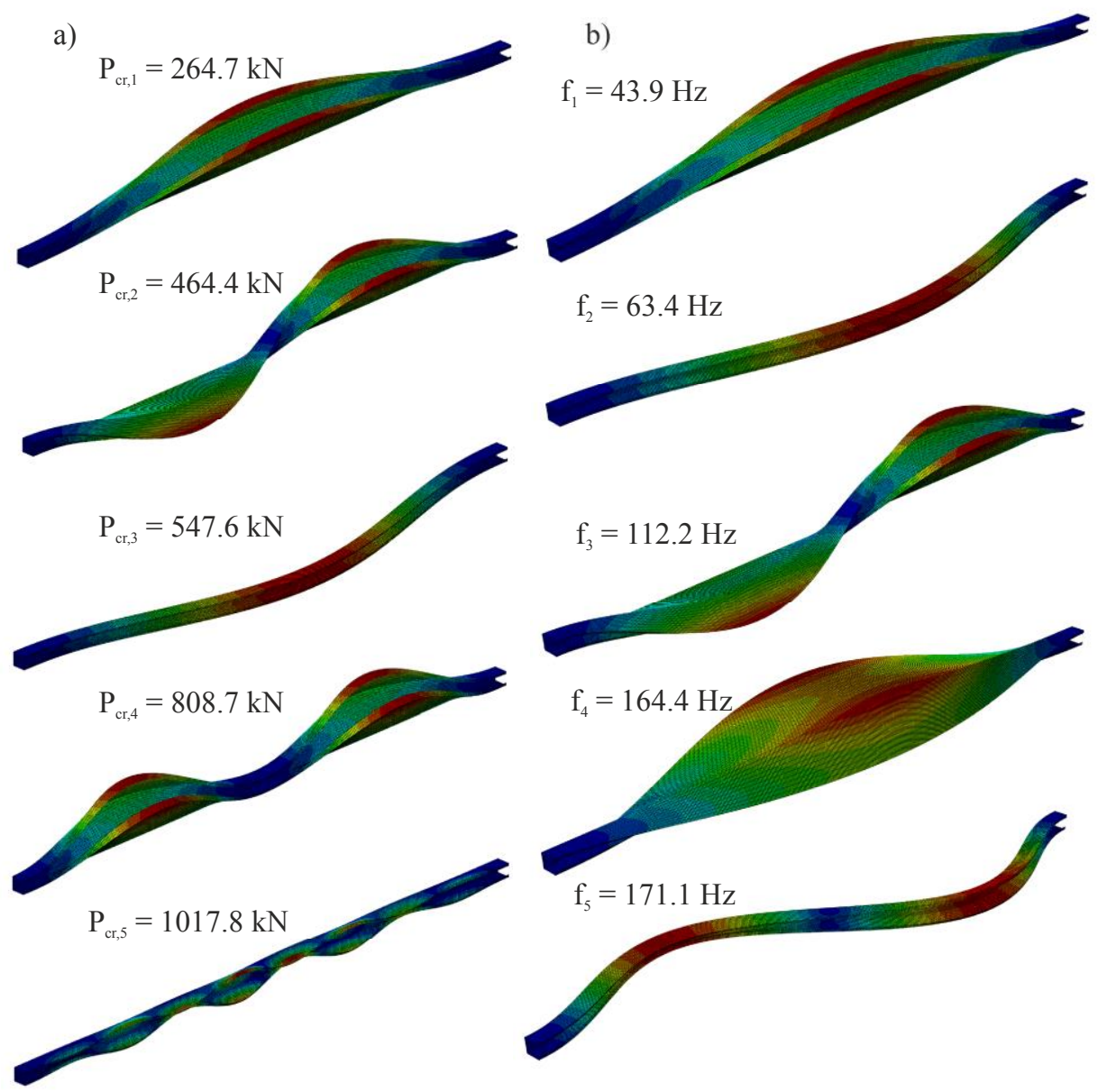

Fig. 7. The primary modes in the column B: linear buckling analysis (a) and dynamic eigenvalue analysis (b).

The first buckling load in the column B equals to $264.7 \mathrm{kN}$. The corresponding buckling mode shows a global, flexural-torsional deformation including a single semi-wave. The second and third buckling loads equal 464.4 and $547.6 \mathrm{kN}$, respectively. The corresponding buckling modes show a flexural-torsional shape with two semi-waves and a global, flexural 
shape, respectively. The first buckling load constitutes $61.5 \%$ of the maximum loadcarrying capacity. It is possibly caused by difficulties to find a limit point because any intended imperfections of the investigated structures are not introduced. The introduction of a slight geometrical imperfection is intended to fix the problem, but the author wanted to analyse perfect column geometries. The deformations received in static non-linear analysis and buckling analysis converge. The primary five buckling modes and their corresponding buckling loads are given in Fig. 7a. The analytical flexural-torsional buckling load equals $266.8 \mathrm{kN}$. The difference between numerical and analytical results is lower than $1 \%$.

The first natural frequency equals $43.86 \mathrm{~Hz}$, the second $-63.37 \mathrm{~Hz}$, the third 112.2 Hz. The primary five vibration modes and their corresponding natural frequencies values are given in Fig. 7b. Both first buckling and vibration modes coincide, but the second and third modes interchange, making it still possible to find buckling-vibration mode equivalents. It is a key issue due to linear relations between the squares of the natural frequencies and the applied load only in the case of convergent buckling and vibration modes.

The correlation between the compressive forces and the squares of the first five natural frequencies was plotted in Fig. 8. Linear relations occur in each case to the point when the applied load becomes the first buckling load. The lowest square of a correlation coefficient is 0.9997 . The first natural frequency drops to zero when the load equals $266 \mathrm{kN}$. While compared to the first buckling load the difference is lower than $0.5 \%$. It is caused by a greater maximum compressive load-carrying capacity compared to the minimum buckling load. Here the post-buckling behaviour is analysed. When the applied load reaches $285 \mathrm{kN}$, the first natural frequency rapidly increases to the new maximum, next declines again. After reaching the first buckling load level the higher order natural frequencies change their performance. The square of the second frequency changes the slope of the curve. The curve corresponding to the square of the third frequency reaches its minimum and rises, whereas the fourth one starts to decrease rapidly. The Vibration Correlation Technique predicts the calculated buckling load sufficiently.

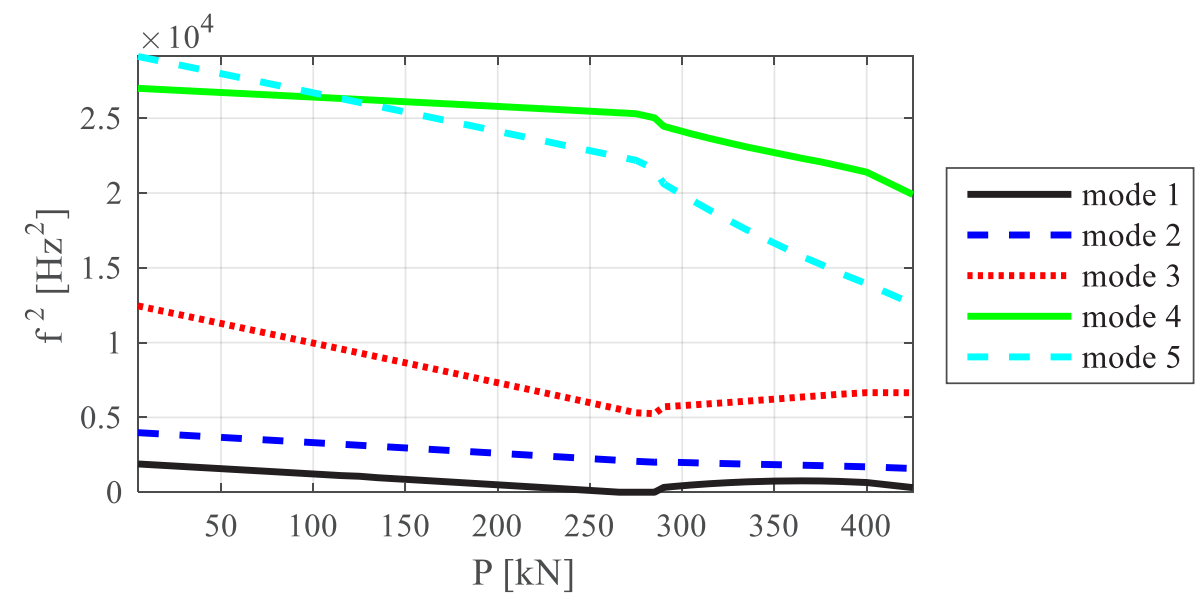

Fig. 8. The relationship between compressive load and squares of natural frequencies in the column B with linearly-elastic material model.

The introduction of plasticity into the analysis did not affect the vibration frequencies and the corresponding modes. The correlation between squares of the natural frequencies and applied load is linear, but it varies when the stresses in the column achieve the yield stress level (see Fig. 9). Therefore, the presented method to predict the buckling load limits 
its use only to the elastic buckling range. While plasticity is the major structural account, the Vibration Correlation Technique fails.

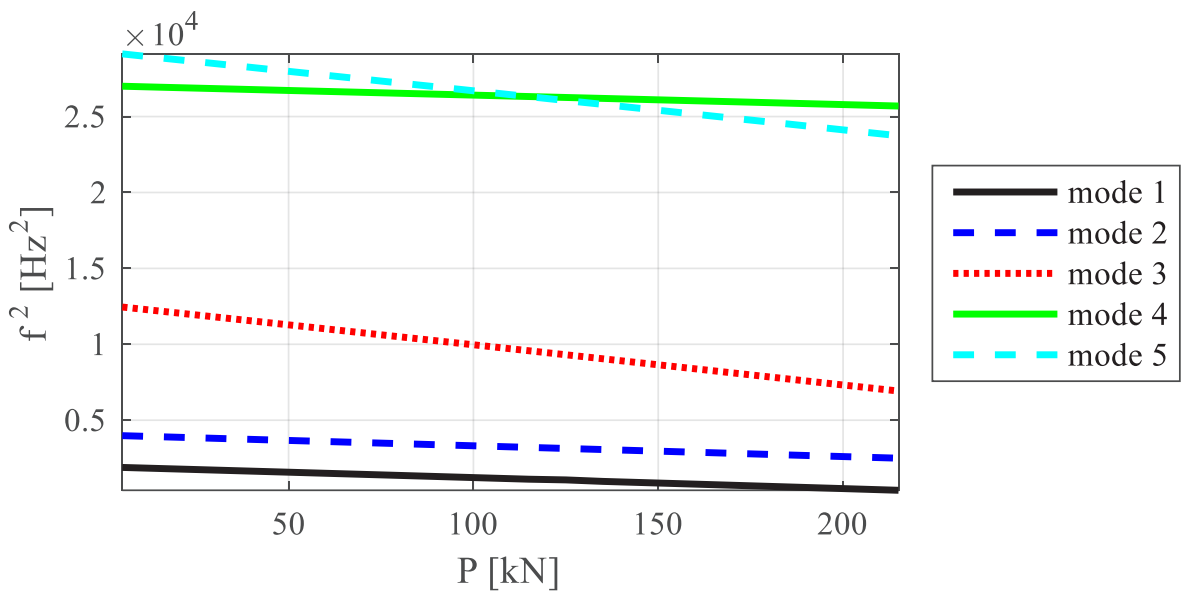

Fig. 9. The relationship between compressive load and squares of natural frequencies in the column B with elastic-perfectly plastic material model.

\section{Conclusions}

The Vibration Correlation Technique can be successfully applied in thin-walled columns exhibiting global - flexural-torsional and flexural - buckling. The effectiveness of the method to estimate the realistic buckling loads is high. Unfortunately, its range is limited to the buckling-prone structures. The method cannot be applied to predict the limit load of the structures; it is limited to elastic buckling loads only, thus the inelastic buckling cases are not dedicated for such estimations.

Local and distortional buckling patterns have not been investigated so far, thus the author is planning further research on such columns. Additionally, physical tests are planned on real thin-walled elements compressed in a testing machine, in order to compare numerical and experimental results.

\section{References}

1. EN 1993-1-3 (2006).

2. J. Singer, J. Arbocz, T. Weller, Buckling experiments: experimental methods in buckling of thin-walled structures: shells, built-up structures and additional topics. Vibration Correlation Techniques (VCT), 2 (John Wiley \& Sons, New York, 2002)

3. M.A. Arbelo, S.F.M. de Almeida, V. Donadon, S.R. Rett, R. Degenhardt, S.G.P. Castro, K. Kalnins, O. Ozolins, THIN WALL STRUCT, 79, 119-128 (2014)

4. Ł. Żmuda-Trzebiatowski, P. Iwicki, Shell Structures: Theory and Applications, 4, 481484 (CRC Press/Balkema, London, 2018)

5. H. Abramovich, D. Govich, A. Grunwald, PROG AEROSP SCI, 78, 62-73 (2015)

6. E. Skukis, O. Ozolins, K. Kalnins, M.A. Arbelo, PROCEDIA ENGINEER, 172, 10231030 (2017)

7. Abaqus 6.12. Theory Manual (Dassault Systemes, 2012) 